\title{
Age-Related Effects on Postural Control under Multi-Task Conditions
}

\author{
Urs Granacher ${ }^{a}$ b Stephanie A. Bridenbaugh ${ }^{c}$ Thomas Muehlbauer ${ }^{a}$ \\ Anja Wehrle $^{c}$ Reto W. Kressig ${ }^{c}$ \\ anstitute of Exercise and Health Sciences, University of Basel, Basel, Switzerland; b Institute of Sport Science, \\ Friedrich-Schiller-University Jena, Jena, Germany; ' Division of Acute Geriatrics, Basel University Hospital, \\ Basel, Switzerland
}

\author{
Key Words \\ Balance $\cdot$ Dual-task $\cdot$ Multi-task $\cdot$ Gait
}

\begin{abstract}
Background: Changes in postural sway and gait patterns due to simultaneously performed cognitive (CI) and/or motor interference (MI) tasks have previously been reported and are associated with an increased risk of falling in older adults. Objective: The objectives of this study were to investigate the effects of a $\mathrm{Cl}$ and/or MI task on static and dynamic postural control in young and elderly subjects, and to find out whether there is an association between measures of static and dynamic postural control while concurrently performing the $\mathrm{Cl}$ and/or MI task. Methods: A total of 36 healthy young ( $n=18$; age: $22.3 \pm 3.0$ years; BMl: $21.0 \pm 1.6 \mathrm{~kg} / \mathrm{m}^{2}$ ) and elderly adults $(n=18$; age: $73.5 \pm 5.5$ years; BMl: $24.2 \pm$ $2.9 \mathrm{~kg} / \mathrm{m}^{2}$ ) participated in this study. Static postural control was measured during bipedal stance, and dynamic postural control was obtained while walking on an instrumented walkway. Results: Irrespective of the task condition, i.e. single-task or multiple tasks, elderly participants showed larger center-of-pressure displacements and greater stride-tostride variability than younger participants. Associations between measures of static and dynamic postural control were found only under the single-task condition in the elderly. Conclusion: Age-related deficits in the postural control system seem to be primarily responsible for the observed results. The weak correlations detected between static and dy-
\end{abstract}

namic measures could indicate that fall-risk assessment should incorporate dynamic measures under multi-task conditions, and that skills like erect standing and walking are independent of each other and may have to be trained complementarily.

Copyright $\odot 2010$ S. Karger AG, Basel

\section{Introduction}

The rate of falls among community-dwelling, healthy elderly people aged 65 and older amounts to $0.3-1.6$ falls per person annually (weighted mean: 0.6 falls). Persons living in long-term care institutions have much higher rates (0.6-3.6 falls per bed annually; mean: 1.7 falls) [1]. Fall-related injuries affect elderly individuals in terms of a poor quality of life caused by restricted mobility and functional decline [2]. Deficits in static and dynamic postural control have been identified as major intrinsic risk factors for falls in old age $[3,4]$.

Postural control can be described as the ability to control the body's position in space for the purpose of balance and orientation [5]. Under static conditions, the base of support remains stationary and only the center of mass moves, whereas under dynamic conditions, both the base of support and the center of mass shift [6]. Dynamic balance control is more challenging because the center of mass remains at distinct phases of the gait cycle (singlelimb support) outside the base of support [6]. Thus, it is

PD Dr. phil. Urs Granacher

Institute of Exercise and Health Sciences, University of Basel Birsstrasse 320B

CH-4052 Basel (Switzerland)

E-Mail karger@karger.ch_Accessible online at:

www.karger.com www.karger.com/ger 
Table 1. Group characteristics

\begin{tabular}{lllll}
\hline & $\begin{array}{l}\text { Age } \\
\text { years }\end{array}$ & $\begin{array}{l}\text { Height } \\
\mathrm{cm}\end{array}$ & $\begin{array}{l}\text { Mass } \\
\mathrm{kg}\end{array}$ & $\begin{array}{l}\mathrm{BMI} \\
\mathrm{kg} / \mathrm{m}^{2}\end{array}$ \\
\hline Young $(\mathrm{n}=18)$ & $22.3 \pm 3.0$ & $172.3 \pm 7.5$ & $62.4 \pm 7.6$ & $21.0 \pm 1.6$ \\
Old $(\mathrm{n}=18)$ & $73.5 \pm 5.5$ & $172.1 \pm 8.0$ & $72.0 \pm 13.0$ & $24.2 \pm 2.9$
\end{tabular}

Values are means $\pm \mathrm{SD}$

not surprising that a large number of falls occur during ambulation in the elderly [7]. In fact, it was reported that slips and trips account for $30-50 \%$ of falls in communitydwelling older adults [8]. Therefore, it has been recommended that primarily measures of dynamic postural control should be applied for the assessment of balance dyscontrol in the elderly. Furthermore, if the assessment is restricted to quiet stance conditions, particularly challenging elements should be incorporated in the testing because normal quiet stance alone may be insensitive to many balance problems [9]. Dual-task experiments represent such a challenge since two tasks (postural plus a secondary cognitive/motor task) are performed simultaneously. In fact, Beauchet et al. [10] proved that the concurrent execution of backward counting while walking had a significant impact on gait variability and, thus, gait instability in young healthy adults. This implies that the control of posture is not, as believed for many years, highly automatic, i.e. regulated on a spinal level by reflexive muscle contractions, but that it requires attentional processing even in young healthy adults. The attentional demand for the control of posture seems to be higher in elderly compared to young adults because interference between concurrent cognitive and static as well as dynamic postural tasks was reported to be greater in old compared to young adults $[11,12]$. Age-related deteriorations in the visual, proprioceptive and vestibular systems [13] seem to be responsible for the increased attentional demand in the elderly to maintain postural stability [14].

Recently, Beauchet et al. [15] reported that fall-risk assessment of postural control under dual-task (DT) conditions has an added value for fall prediction compared to assessment protocols conducted under the single-task (ST) condition. However, in everyday life, interference may not only occur between cognitive (e.g. talking to somebody) and postural control tasks (e.g. standing, walking), but also between motor (e.g. carrying grocery bags) and postural control tasks (e.g. standing, walking). Yet, there is only sparse information in the literature re- garding this issue [16]. Furthermore, it could not yet have been elucidated conclusively whether an assessment of postural control should be conducted under dynamic conditions only, as indicated by potential functional implications, or whether static assessment protocols might be of similar value. In fact, an advantage of static over dynamic assessment might be the relatively easy-to-administer testing protocol for practitioners and therapists.

Therefore, the objectives of this study were to investigate the effects of a cognitive (CI) and/or motor interference (MI) task on static and dynamic postural control in young and elderly subjects, and to find out whether there is an association between static and dynamic postural control measures while concurrently performing the CI and/or MI task. It is hypothesized that postural sway and stride-to-stride variability are impaired in old compared to young subjects in all task conditions. Furthermore, it is expected that increasing task complexity (from ST to multi-task) results in respective increments in postural sway and stride-to-stride variability. This increment should be more prominent in elderly compared to young subjects. Since there is evidence of an association between quiet-stance behavior and dynamic postural control in healthy young and elderly adults, indicating that similar neuromuscular mechanisms regulate static and dynamic posture [17], it is hypothesized that there is an association between these balance capacities. The present findings could bridge the gap between research on balance in older adults and its application to clinical practice. In fact, clinical assessment would be simplified if a meaningful association between measures of static and dynamic postural control could be observed.

\section{Subjects and Methods}

\section{Participants}

Thirty-six healthy young $(\mathrm{n}=18)$ and elderly $(\mathrm{n}=18)$ community-dwelling participants gave their written informed consent to participate in this study (table 1). The participants were healthy with no previous lower extremity trauma and no history of serious muscular, neurological, cardiovascular, metabolic and inflammatory diseases. The participants were capable of walking independently without any assistive device, and they had no prior experience with the applied tests. The study was approved by the ethics committee of the University of Basel, and all experiments were conducted according to the Declaration of Helsinki.

\section{Apparatus}

Measurements were carried out in our gait laboratory and included testing of static postural control on a balance platform and the analysis of dynamic postural control while walking on a pressure-sensitive 10-meter walkway. Test circumstances (e.g. room 
illumination, temperature, noise) were in accordance with recommendations for posturographic testing [18].

\section{Stance}

A balance platform (HUR BT4A; Hur Labs, Tampere, Finland) was used for the assessment of static postural control, which consisted of 4 sensors measuring total displacements of the center of pressure (COP) in centimeters. Data were acquired for $30 \mathrm{~s}$ at a sampling rate of $80 \mathrm{~Hz}$ [18]. Total displacement of the COP was computed. The intraclass correlation coefficients (ICC) for COP displacement ranged from 0.77 to 0.94 for the different task conditions.

Walk

Dynamic postural control was determined during steady-state walking on a 10 -meter walkway using the GAITRite ${ }^{\circledR}$ system (CIR Systems Inc., Havertown, Pa., USA). The distribution of pressure during walking was monitored at $80 \mathrm{~Hz}$, enabling spatial and temporal gait data to be collected. Besser et al. [19] reported that $5-8$ strides are necessary for $90 \%$ of the individuals tested by GAITRite instrumentation to obtain reliable mean estimates of spatiotemporal gait parameters. Temporal and spatial parameters of gait seem to be important in the assessment of gait instability in community-dwelling elderly adults [20]. In a first step, means and SD of stride time, stride length and stride velocity were computed. Stride time was defined as the time (in seconds) between the first contacts of 2 consecutive footfalls of the same foot. Stride length was defined as the linear distance (in centimeters) between successive heel contacts of the same foot. Additionally, stride velocity (in centimeters per second) was calculated as stride length divided by stride time. Coefficients of variation (CV) were calculated for the abovementioned parameters according to the formula $[(\mathrm{SD} /$ mean $) \times 100][21]$ and used as outcome measures. The ICC for our gait parameters ranged from 0.79 to 0.98 for the different task conditions.

\section{Questionnaire}

The Mini-Mental State Examination (MMSE) was applied, which is a valid test of cognitive function. It separates patients with cognitive disturbance from those without such disturbance. The test-retest reliability of the MMSE is high with $\mathrm{r}=0.89$. Crosscorrelation with the Wechsler Adult Intelligence Scale score revealed a correlation coefficient of $r=0.78$ [22]. According to Folstein et al. [22], an MMSE total score of less than 20 separates patients with dementia or functional psychosis from normal participants and those with anxiety neurosis or personality disorder.

The Falls Efficacy Scale-International (FES-I) was developed for the documentation of fall-related self-efficacy in older persons. The FES-I showed excellent internal and test-retest reliability (Cronbach's $\alpha=0.96$; ICC $=0.96$ ). In addition, the FES-I has been shown to have acceptable construct validity in different samples in different countries (range: $r=0.79-0.82$ ) [23].

\section{Testing}

Upon entering the gait laboratory, participants were asked to answer the questions of the MMSE and the FES-I. Before testing, a trained evaluator gave standardized verbal instructions regarding the test procedure with a visual demonstration of the bipedal stance position and the walking test. Thereafter, static and dynamic postural control was measured under ST, DT and triple-
Table 2. Summary of experimental procedure

\begin{tabular}{lll}
\hline & Task & Condition \\
\hline \multirow{2}{*}{ tatic postural control } & standing & ST \\
& standing + CI & DT \\
& standing + MI & DT \\
& standing + CI + MI & TT \\
\hline Dynamic postural control & walking & ST \\
& walking + CI & DT \\
& walking + MI & DT \\
& walking + CI + MI & TT \\
\hline
\end{tabular}

task (TT) conditions. ST refers to standing/walking alone, DT to standing/walking with a CI or MI task, and TT to standing/walking with CI and MI tasks. During the measurement of static postural control, the participants stood barefooted on both legs (heels together at an angle of $30^{\circ}$ between the medial sides of the feet) and in an upright position [18] for $30 \mathrm{~s}$ on a balance pad (Airex Balance Pad; Gaugler \& Lutz, Aalen-Ebnat, Germany) which covered the balance platform. The participants were asked to place their hands on the hips and to look straight ahead, focusing on a circular area attached to the wall. They were instructed to remain as stable as possible and to refrain from any voluntary movements during the trials. To assess dynamic postural control, they walked with their own footwear at a self-selected speed, initiating and terminating each walk a minimum of $1 \mathrm{~m}$ before and after the 10 -meter walkway to allow sufficient distance to accelerate to and decelerate from a steady-state of ambulation across the walkway.

Static as well as dynamic postural control was also examined while performing a concurrent attention-demanding CI and/or MI task. The CI task was an arithmetic task in which the participants loudly recited serial subtractions by 3 , starting from a randomly selected number between 300 and 900 given by the experimenter [24]. The MI task required participants to hold 2 interlocked sticks steadily in front of their body. One stick was held in each hand, with the elbow in 90-degree flexion. Each stick had a ring at the end with a diameter of $4 \mathrm{~cm}$, and the rings were interlocked [16]. The participants were advised not to let the rings touch each other. When the DT methodology was used, participants were instructed to give equal priority to both tasks in order to create real-life conditions [25]. In addition, recent results indicate that task prioritization had no effect on measures of postural control while dual-tasking [26]. A summary of the experimental procedure is presented in table 2 . All tests were performed in a counterbalanced order for static and dynamic postural control conditions separately. Since we were interested in the potential effects of the additional CI and MI tasks on changes in gait dynamics and displacements of COP, and were not primarily concerned with the performance of participants in the CI and MI tasks itself, we focused on the results of the postural control measures.

\section{Statistical Analysis}

An a priori power analysis [27] with an assumed type I error of 0.05 and a type II error rate of 0.10 (statistical power $=0.90$ ) was conducted for the parameter stride time CV [16] and revealed that 


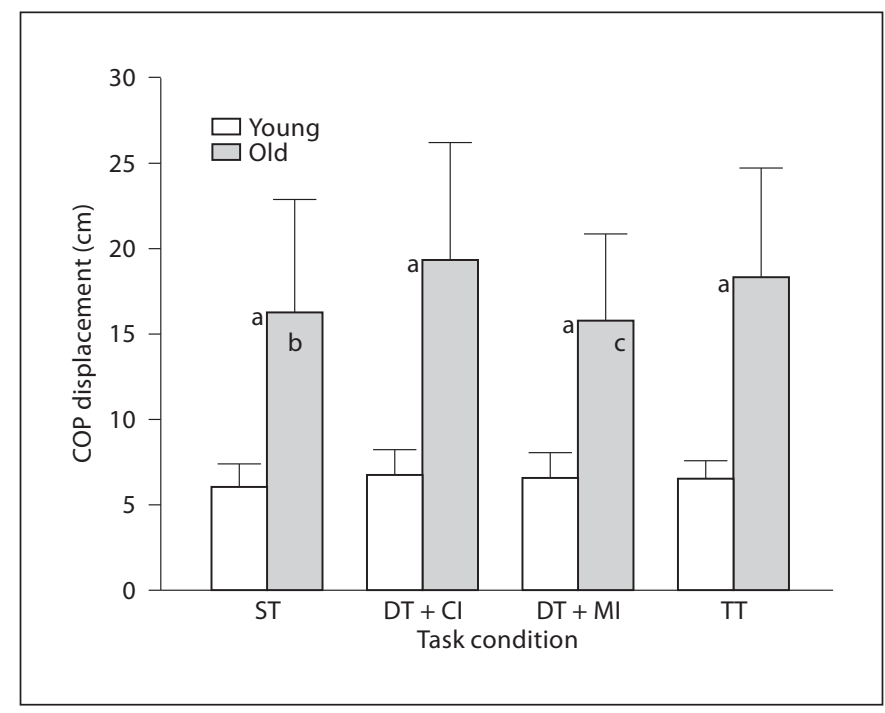

Fig. 1. COP displacements for younger and older participants by task condition. ${ }^{\text {a }}$ Significant differences between younger and older adults; ${ }^{\mathrm{b}}$ significant differences between ST and DT $+\mathrm{CI} ;{ }^{\mathrm{c}}$ significant differences between DT + MI and TT.

18 persons per group would be sufficient for finding statistically significant age-related effects. A 2 (group: young, old) $\times 4$ (task: ST, DT + CI, DT + MI, TT) analysis of variance with repeated measures of tasks was performed to examine differences between the 2 age groups and between the 4 task conditions for COP displacements and for stride-to-stride variability in stride time, stride length and stride velocity. Post hoc tests with the Bonferroni-adjusted $\alpha$ were conducted to identify the comparisons that were statistically significant. Associations between COP displacements and stride-to-stride variability measures were assessed using Pearson's correlation. Associations are reported by their correlation coefficient and their level of significance. All analyses were performed using the Statistical Package for the Social Sciences (SPSS), version 16.0. The significance level was set at $\mathrm{p}<0.05$.

\section{Results}

The results of the MMSE (mean: $27.6 \pm 2.3$; range: 22-30) and the FES-I (mean: $19.5 \pm 2.3$; range: 17-25) indicate that the elderly participants in this study were cognitively healthy without any serious concern about falling.

\section{Stance}

COP Displacements. Irrespective of the task condition, elderly participants showed larger COP displacements than the younger participants (fig. 1). The analysis indicated main effects of group $[\mathrm{F}(1,34)=60.80 ; \mathrm{p}<0.01$; $\left.\eta^{2}=0.64\right]$ and task $\left[\mathrm{F}(3,210)=7.64 ; \mathrm{p}<0.01 ; \eta^{2}=0.18\right]$.
The analysis also detected a group $\times$ task interaction $[\mathrm{F}(3$, $210)=5.08 ; p<0.01 ; \eta^{2}=0.13$ ]. Young participants were able to effectively compensate for increased task complexity with virtually no changes in COP displacements. COP displacements in older participants were significantly shorter in the ST condition than in conjunction with serial subtraction $(\mathrm{p}<0.05)$, and performance in the $\mathrm{DT}+$ MI was significantly better compared to the TT $(\mathrm{p}<0.01)$. In addition, younger adults performed significantly better than older adults in all task conditions $(\mathrm{p}<0.001)$.

\section{Walk}

Stride Time CV. Older adults walked with larger stride time variability than the younger adults (fig. 2a). The analysis indicated main effects of group $[\mathrm{F}(1,34)=13.64$; $\left.\mathrm{p}<0.01 ; \eta^{2}=0.29\right]$ and task $[\mathrm{F}(3,210)=7.03 ; \mathrm{p}<0.01$; $\left.\eta^{2}=0.17\right]$. The analysis also detected a group $\times$ task interaction $\left[\mathrm{F}(3,210)=4.09 ; \mathrm{p}<0.01 ; \eta^{2}=0.11\right]$. In both age groups, variability in the ST condition was significantly smaller than in the TT condition $(p<0.05)$. In older adults, this was also true for the comparison of the ST with the DT + MI condition ( $\mathrm{p}<0.01)$. Younger compared to older adults showed less stride time variability in the DT + MI $(\mathrm{p}=0.001)$ and TT conditions $(\mathrm{p}=0.003)$.

Stride Length $C V$. The stride length variability in the older participants was greater compared to the younger adults (fig. 2b). The analysis indicated main effects of group $\left[\mathrm{F}(1,34)=54.98 ; \mathrm{p}<0.01 ; \eta^{2}=0.62\right]$ and task $\left[\mathrm{F}(3,210)=15.68 ; \mathrm{p}<0.01 ; \eta^{2}=0.32\right]$. The analysis also detected a group $\times$ task interaction $[\mathrm{F}(3,210)=5.05$; $\mathrm{p}<0.01 ; \eta^{2}=0.13$ ]. Both ages performed significantly less variably in the ST condition compared to the DT + MI (both $\mathrm{p} \leq 0.01$ ) and TT conditions (both $\mathrm{p} \leq 0.01$ ). Additionally, younger adults performed significantly better than older adults, irrespective of the task condition ( $\mathrm{p} \leq$ $0.001)$.

Stride Velocity $C V$. The younger adults walked with less stride velocity variability than the older adults (fig. 2c). The analysis indicated main effects of group $\left[\mathrm{F}(1,34)=14.99 ; \mathrm{p}<0.01 ; \eta^{2}=0.31\right]$ and task $[\mathrm{F}(3,210)=$ 8.87; $\left.\mathrm{p}<0.01 ; \eta^{2}=0.21\right]$. The analysis also detected a group $\times$ task interaction $\left[\mathrm{F}(3,210)=3.92 ; \mathrm{p}<0.05 ; \eta^{2}=\right.$ $0.10]$. As in stride length variability, younger and older adults walked with significantly less stride velocity variability in the ST condition compared to the DT + MI (both $\mathrm{p}<0.01$ ) and TT conditions (both $\mathrm{p} \leq 0.01$ ). With the exception of the DT + CI condition, younger compared to older adults showed less stride velocity variability in all task conditions ( $\mathrm{p} \leq 0.007$ ). 


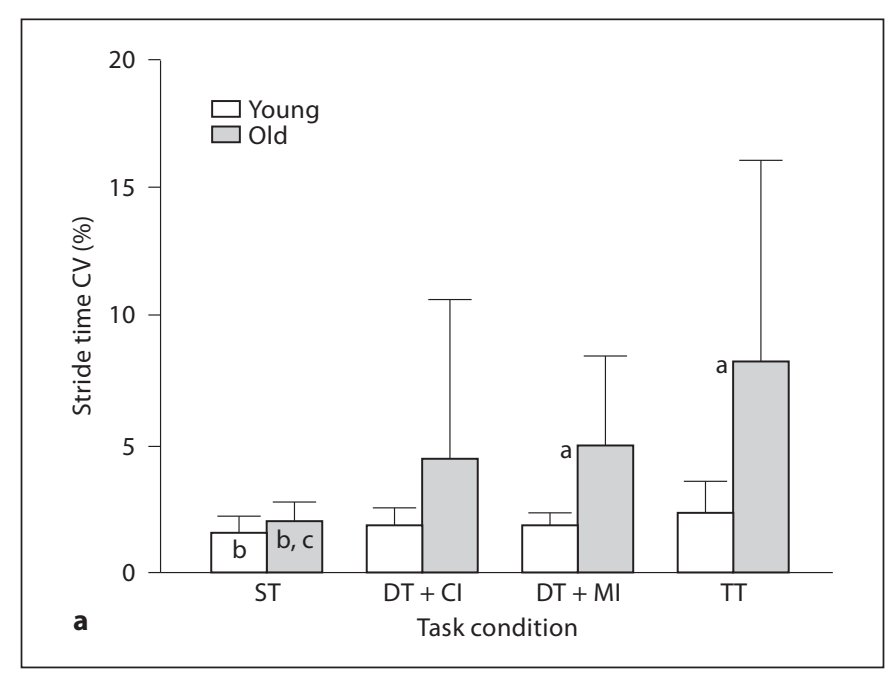

Fig. 2. Stride-to-stride variability in stride time (a), stride length (b) and stride velocity (c) for younger and older participants by task condition. ${ }^{\text {a }}$ Significant differences between younger and older adults; ${ }^{\mathrm{b}}$ significant differences between ST and TT; ${ }^{\mathrm{c}}$ significant differences between ST and DT + MI.

Associations between COP Displacements and Strideto-Stride Variability. A statistically significant relationship between COP displacements and stride length/velocity CV was found in the elderly participants under ST conditions only. Regarding the young participants, no statistically significant associations between COP displacement and temporal and spatial stride-to-stride variability were detected, irrespective of the task condition considered (table 3).

\section{Discussion}

The main findings of this study were: (1) the simultaneous performance of quiet stance or walking with a CI and/or MI task resulted in increased COP displacements
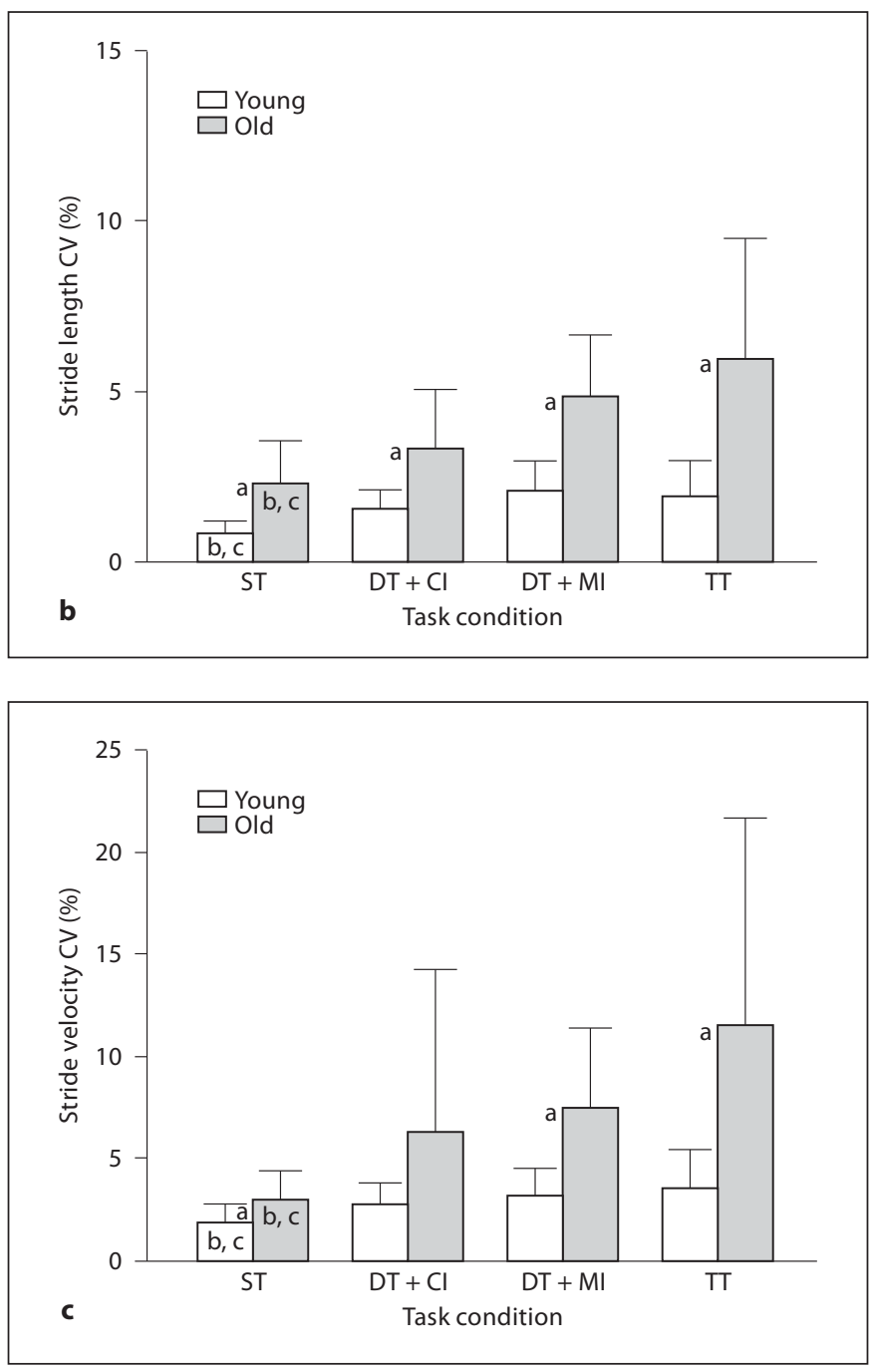

and greater stride-to-stride variability in old compared to young adults irrespective of the task condition; (2) in the elderly, COP displacements increased with progression of task complexity; (3) in both age groups, stride-to-stride variability during the ST condition was significantly smaller than during the TT condition, and (4) associations between COP displacement and stride length/velocity CV were only present in the elderly adults under ST conditions.

The findings are in accordance with the literature regarding the effects of CI tasks on postural sway in older adults. Melzer et al. [28] found increased postural sway in old compared to young subjects when performing ST and DT tests. Similar results were reported for older adults with and without a history of falls [29]. 
Table 3. Correlation between COP displacements and stride-tostride variability measures

\begin{tabular}{lrrrr}
\hline \multicolumn{4}{c}{ COP displacements } \\
\cline { 2 - 5 } & ST & DT + CI & DT + MI & TT \\
\hline Young (n= 18) & & & & \\
Stride time CV & -0.02 & -0.26 & -0.22 & 0.11 \\
Stride length CV & -0.17 & 0.24 & 0.15 & 0.24 \\
Stride velocity CV & 0.05 & -0.05 & -0.11 & 0.09 \\
Old (n = 18) & & & & \\
Stride time CV & 0.21 & -0.41 & -0.28 & -0.37 \\
Stride length CV & $0.51^{*}$ & -0.30 & -0.38 & -0.10 \\
Stride velocity CV & $0.56^{*}$ & -0.42 & -0.31 & -0.36 \\
\hline
\end{tabular}

${ }^{*} \mathrm{p}<0.05$.

Furthermore, the results of the present study concerning the impact of a CI task on stride-to-stride variability are in agreement with a recently conducted study. Hollman et al. [30] reported a greater variability in stride velocity during DT walking compared to normal walking in middle-aged and older adults. In addition, the variability in stride velocity was increased in both walking conditions in older compared to middle-aged and younger adults [30]. Similar results were found in a later study comparing young with elderly women regarding the variability in stride velocity [31]. In another study, Laessoe et al. [16] investigated the effects of a CI and/or MI task in young and elderly community-dwelling subjects on stride time variability while walking along a figure of eight at a self-selected speed. The authors reported that the elderly participants had increased stride time variability when normal walking was compared to walking with a CI task, and walking with both a CI and an MI task. Our findings are in accordance with the findings by Laessoe et al. [16] regarding an increased stride-to-stride variability with increasing task complexity in elderly participants. However, in contrast to our results, Laessoe et al. [16] did not detect differences between tasks in the young adults. This discrepancy cannot be explained by differences in the severity of CI and MI tasks between the two studies because the arithmetic task was even more difficult in the study conducted by Laessoe et al. [16] (serial subtractions by 7 vs. subtractions by 3 in our study), and the applied MI task was similar in the two studies. Furthermore, walking in a figure of eight seems to be more challenging than walking straight. Thus, the severity of the walking condition cannot account for differences either. It is suggested though that the observed discrepancy can most likely be explained by the validity and sensitivity of the testing apparatus applied. GAITRite instrumentation has been reported to have high reliability (ICC $>0.85$ ) and high concurrent validity (ICC >0.93) when compared with videobased motion analysis systems for spatial and temporal parameters of gait [32, 33]. Laessoe et al. [16] used forcesensing resistors, which were placed under each heel of the participants to record step and stride times. There were no references made regarding the validity and reliability of the foot switches. Thus, due to the higher sensitivity of the GAITRite system, it might be possible that we were able to detect task differences in young adults, whereas Laessoe et al. [16] were not.

What could be the underlying reasons for the age-related effects seen in static and dynamic postural control measures under ST, and particularly under DT and TT conditions? Age-related impairments occur at every stage of the postural control system and may therefore account for the deficits in COP displacements and strideto-stride variability observed in old compared to young subjects. In terms of input, there is a deterioration of the peripheral sensory processes (visual, proprioceptive and vestibular) [13]. Central integrative processes are impaired in the elderly in terms of changes in presynaptic inhibition [34] and a decline in the number of interneurons [35]. On the output side, slowing of peripheral nerve conduction velocity, a reduction in the number of $\alpha$-motoneurons and a decline in muscle mass have been demonstrated in elderly people [36]. With the loss of visual, proprioceptive and vestibular sensitivity in old age, more of the attention of elderly subjects is required to maintain stance/gait stability, particularly in more highly demanding situations.

For many years, walking has been considered a highly automated movement which is performed with a minimum of conscious control [37]. Studies applying DT or TT paradigms proved that the competition between the attentional demands required for gait and the concurrent performance of a CI or MI task are responsible for gait alterations $[12,16]$. For example, if two tasks are concurrently conducted, with the primary task comprising postural control and the secondary task cognitive or motor information processing, a decrement in performance of one or both tasks can be observed due to the brain's limited information processing capacity ('central overload') [38]. Less resources are required for relatively undemanding postural tasks such as sitting or standing on stable surfaces, whereas attentional demands are increased when individuals are walking $[39,40]$. The most com- 
monly used explanation for posture-cognition DT interference is that these tasks compete in parallel for one or more resource pools ('crosstalk' or 'neural structure theory'), or else serially engage common input/output mechanisms ('bottleneck theory') [38, 41]. The latter refers to the idea that two concurrently performed tasks which require a certain cognitive processing operation at the same point in time can only be carried out sequentially [38].

In the present study, associations between COP displacements and stride length/velocity CV were only present in elderly adults under ST conditions. Other studies found similar results regarding this matter. Shimada et al. [42] investigated static (sensory organization test) and dynamic postural control (decelerating perturbation impulse while walking on a treadmill) in young and elderly adults. A weak correlation was found in the elderly subjects for postural sway measured under conditions of inaccurate visual and somatosensory input and latency, which was analyzed from the onset of treadmill perturbation to the point of maximum posterior trunk acceleration. In contrast to the results by Shimada et al. [42] and our results, Hsiao-Wecksler et al. [17] observed an association between COP displacements during quiet stance and COP displacements during mild perturbation. The authors concluded that it is possible to predict the dynamic postural control response from quiet-stance behavior in elderly adults. Therefore, Hsiao-Wecksler et al. [17] suggested that the postural control system may use the same control mechanisms during quiet stance and mild perturbation conditions. The reason for the discrepancy between the results by Shimada et al. [42], our results and the findings by Hsiao-Wecksler et al. [17] can most likely be found in the different methods applied in these studies. In the study by Shimada et al. [42] and in the present study, associations between quiet stance measures and measures of gait perturbation or gait variability were investigated. However, Hsiao-Wecksler et al. [17] examined the relationship between measures of quiet stance and measures of mild perturbation during stance. The authors reported that the compensation of these perturbation impulses did not even force subjects to take a step for the maintenance of balance. Thus, it can be speculated that, in fact, different neuromuscular mechanisms might be responsible for the regulation of a primarily static postural control task (e.g. mild stance perturbation) and a dynamic postural control task (e.g. walking, gait perturbation). This could be a potential reason why an association was found in the first case [17] but not in the last [42]. In addition, Hsiao-Wecksler et al. [17] as well as Shimada et al. [42] conducted their postural control mea-

Age-Related Balance Performance sures under the ST condition only, whereas we performed postural tasks under multi-task conditions. Thus, it could be hypothesized that in the elderly, it is not the postural skill itself (static vs. dynamic) but the task condition (single vs. multi) that may account for the absence/presence of associations in postural control measures. In other words, compared to standing/walking alone, the inclusion of an additional task requires modified postural control due to increased attentional demand. For example, standing while concurrently performing a CI and/or MI task results in better performance than DT/TT walking, which is supported by the observed negative correlations. During standing, the center of mass remains stable over the base of support, whereas during walking it is constantly shifted toward the edge of the base of support, resulting in increased postural demands.

These preliminary results may have functional implications for future directions in fall-risk assessment as well as in planning and developing adequate training programs for counteracting intrinsic fall-risk factors in the elderly. Based on our findings, it can be hypothesized that different neuromuscular mechanisms are responsible for the regulation of static and dynamic postural control, particularly under multi-task conditions. Given that falls primarily occur during walking and not during quiet stance in the elderly [7], fall-risk assessment should particularly be carried out under multi-task dynamic conditions to identify potential balance problems. From a fallpreventive point of view, our results could indicate that erect standing and walking are independent of each other and may have to be trained complementarily under multi-task conditions. In fact, this is reinforced by a study which documents that balance skills developed through training that involves standing tasks have limited effects on balance control during walking in hemiparetic patients [43].

We acknowledge that this study has some limitations that warrant discussion. First, due to the cross-sectional character of this study, a causal relationship between the age of participants and the findings observed regarding measures of static and dynamic postural control under ST, DT and TT conditions cannot be drawn. Therefore, care is needed when generalizing the present findings to other kinds of testing situations or groups of subjects. Second, our participants were balance tested in bipedal stance on a balance platform with a balance pad on top. When planning our balance test setup, we were aware of a possible 'ceiling effect' (i.e. COP displacements cannot take on a value higher than some limit due to the lack of severity of the balance test), particularly in the young 
adults. However, results from pilot testing clearly indicated that one-legged stance was too difficult for most of the seniors tested. This is why we included a balance pad to increase task severity. Third, we only reported results regarding total COP displacements as a global measure for static postural control. Our initial analysis included velocity, range, root mean square and the $\mathrm{CV}$ for total $\mathrm{COP}$ displacements as well as for COP displacements in mediolateral and anterior-posterior direction. Since the statistical analysis revealed no additional information by the integration of the abovementioned parameters, we decided to focus on the global parameter of COP displacements only.

\section{Conclusions}

In summary, irrespective of the task condition, elderly participants showed larger COP displacements and greater stride-to-stride variability than the younger adults. In the elderly, COP displacements increased with progression of task complexity. In both age groups, stride-tostride variability during the ST condition was significantly smaller than during the TT condition. These results indicate increased stance and gait instability during the concurrent performance of attention-demanding tasks that are probably due to age-related deteriorations in the postural control system and the inability to allocate attention properly between steady-state standing/walking and a CI and/or MI task. Overall, due to the weak correlations between static and dynamic measures, it can be argued that an assessment of risk of falling should rather focus on dynamic than static measures, which should preferably be conducted under multi-task conditions. In addition, based on our results, skills like erect standing and walking are independent of each other and may have to be trained complementarily under multitask conditions. In fact, recent studies indicated that DT balance training is more effective than ST balance training $[44,45]$.

\section{References}

$\checkmark 1$ Rubenstein LZ: Falls in older people: epidemiology, risk factors and strategies for prevention. Age Ageing 2006;35(suppl 2):ii37ii 41 .

2 Tideiksaar R: Preventing falls: how to identify risk factors, reduce complications. Geriatrics 1996;51:43-55.

3 Maki BE, Holliday PJ, Topper AK: A prospective study of postural balance and risk of falling in an ambulatory and independent elderly population. J Gerontol A Biol Sci Med Sci 1994;49:M72-M84.

-4 Verghese J, Buschke H, Viola L, Katz M, Hall C, Kuslansky G, Lipton R: Validity of divided attention tasks in predicting falls in older individuals: a preliminary study. J Am Geriatr Soc 2002;50:1572-1576.

5 Shumway-Cook A, Woollacott M: Motor Control. Theory and Practical Applications. Philadelphia, Lippincott Williams and Wilkins, 2001.

6 Woollacott MH, Tang PF: Balance control during walking in the older adult: research and its implications. Phys Ther 1997;77:646660.

7 Talbot LA, Musiol RJ, Witham EK, Metter EJ: Falls in young, middle-aged and older community-dwelling adults: perceived cause, environmental factors and injury. BMC Public Health 2005;5:86.
8 Lord SR, Ward JA, Williams P, Anstey KJ: An epidemiological study of falls in older community-dwelling women: the Randwick falls and fractures study. Aust J Public Health 1993; 17:240-245.

$>9$ Patla AE, Frank JS, Winter DA: Balance control in the elderly: implications for clinical assessment and rehabilitation. Can J Public Health 1992;83(suppl 2):S29-S33.

10 Beauchet O, Dubost V, Herrmann FR, Kres sig RW: Stride-to-stride variability while backward counting among healthy young adults. J Neuroeng Rehabil 2005;2:26.

11 Maylor EA, Allison S, Wing AM: Effects of spatial and nonspatial cognitive activity on postural stability. Br J Psychol 2001;92(pt 2):319-338.

12 Dubost V, Kressig RW, Gonthier R, Herrmann FR, Aminian K, Najafi B, Beauchet O: Relationships between dual-task-related changes in stride velocity and stride time variability in healthy older adults. Hum Mov Sci 2006;25:372-382.

13 Shaffer SW, Harrison AL: Aging of the somatosensory system: a translational perspective. Phys Ther 2007;87:193-207.

14 Brown LA, Shumway-Cook A, Woollacott $\mathrm{MH}$ : Attentional demands and postural recovery: the effects of aging. J Gerontol A Biol Sci Med Sci 1999;54:M165-M171.

-15 Beauchet O, Annweiler C, Dubost V, Allali G, Kressig RW, Bridenbaugh S, Berrut G, Assal F, Herrmann FR: Stops walking when talking: a predictor of falls in older adults? Eur J Neurol 2009;16:786-795.
16 Laessoe U, Hoeck HC, Simonsen O, Voigt M: Residual attentional capacity amongst young and elderly during dual and triple task walking. Hum Mov Sci 2008;27:496-512.

17 Hsiao-Wecksler ET, Katdare K, Matson J, Liu W, Lipsitz LA, Collins JJ: Predicting the dynamic postural control response from quietstance behavior in elderly adults. J Biomech 2003;36:1327-1333.

18 Kapteyn TS, Bles W, Njiokiktjien CJ, Kodde $\mathrm{L}$, Massen CH, Mol JM: Standardization in platform stabilometry being a part of posturography. Agressologie 1983;24:321-326.

19 Besser MP, Kmieczak K, Schwartz L, Snyderman M, Wasko J, Selby-Silverstein L: Representation of temporal spatial gait parameters using means in adults without impairment. Gait Posture 1999;9:113.

20 Hausdorff JM, Edelberg HK, Mitchell SL, Goldberger AL, Wei JY: Increased gait unsteadiness in community-dwelling elderly fallers. Arch Phys Med Rehabil 1997;78:278283.

21 Kressig RW, Beauchet O: Guidelines for clinical applications of spatio-temporal gait analysis in older adults. Aging Clin Exp Res 2006;18:174-176

22 Folstein MF, Folstein SE, McHugh PR: 'Mini-Mental State': a practical method for grading the cognitive state of patients for the clinician. J Psychiatr Res 1975;12:189-198. 
-23 Kempen GI, Todd CJ, van Haastregt JC, Zijlstra GA, Beyer N, Freiberger E, Hauer KA, Piot-Ziegler C, Yardley L: Cross-cultural validation of the Falls Efficacy Scale International (FES-I) in older people: results from Germany, the Netherlands and the UK were satisfactory. Disabil Rehabil 2007;29:155162.

24 Pellecchia GL: Dual-task training reduces impact of cognitive task on postural sway. J Mot Behav 2005;37:239-246.

-25 Beauchet O, Dubost V, Aminian K, Gonthier R, Kressig RW: Dual-task-related gait changes in the elderly: does the type of cognitive task matter? J Mot Behav 2005;37:259-264.

-26 Wehrle A, Granacher U, Mühlbauer T: Effect of task prioritization on postural control while dual-tasking. Z Sportpsychol 2010;17: 29-35.

-27 Faul F, Erdfelder E, Lang AG, Buchner A: $G^{*}$ Power 3: a flexible statistical power analysis program for the social, behavioral, and biomedical sciences. Behav Res Methods 2007;39:175-191.

28 Melzer I, Benjuya N, Kaplanski J: Age-related changes of postural control: effect of cognitive tasks. Gerontology 2001;47:189-194.

-29 Shumway-Cook A, Woollacott M, Kerns KA, Baldwin M: The effects of two types of cognitive tasks on postural stability in older adults with and without a history of falls. J Gerontol A Biol Sci Med Sci 1997;52:M232-M240.

- 30 Hollman JH, Kovash FM, Kubik JJ, Linbo RA: Age-related differences in spatiotemporal markers of gait stability during dual-task walking. Gait Posture 2007;26:113-119.
Priest AW, Salamon KB, Hollman JH: Agerelated differences in dual-task walking: a cross-sectional study. J Neuroeng Rehabil 2008;5:29.

32 McDonough AL, Batavia M, Chen FC, Kwon S, Ziai J: The validity and reliability of the GAITRite system's measurements: a preliminary evaluation. Arch Phys Med Rehabil 2001;82:419-425.

33 Bilney B, Morris M, Webster K: Concurrent related validity of the GAITRite walkway system for quantification of the spatial and temporal parameters of gait. Gait Posture 2003; 17:68-74.

34 Earles D, Vardaxis V, Koceja D: Regulation of motor output between young and elderly subjects. Clin Neurophysiol 2001;112:12731279.

35 Terao S, Sobue G, Hashizume Y, Li M, Inagaki T, Mitsuma T: Age-related changes in human spinal ventral horn cells with special reference to the loss of small neurons in the intermediate zone: a quantitative analysis. Acta Neuropathol 1996;92:109-114.

36 McNeil CJ, Doherty TJ, Stashuk DW, Rice CL: Motor unit number estimates in the tibialis anterior muscle of young, old, and very old men. Muscle Nerve 2005;31:461-467.

37 Schubert M, Curt A, Jensen L, Dietz V: Corticospinal input in human gait: modulation of magnetically evoked motor responses. Exp Brain Res 1997;115:234-246.

8 Pashler H: Dual-task interference in simple tasks: data and theory. Psychol Bull 1994;116: 220-244.
39 Lavoie BA, Cody FW, Capaday C: Cortical control of human soleus muscle during volitional and postural activities studied using focal magnetic stimulation. Exp Brain Res 1995;103:97-107.

40 Solopova IA, Kazennikov OV, Deniskina NB, Levik YS, Ivanenko YP: Postural instability enhances motor responses to transcranial magnetic stimulation in humans. Neurosci Lett 2003;337:25-28.

41 Fraizer EV, Mitra S: Methodological and interpretive issues in posture-cognition dualtasking in upright stance. Gait Posture 2008; 27:271-279.

42 Shimada H, Obuchi S, Kamide N, Shiba Y, Okamoto M, Kakurai S: Relationship with dynamic balance function during standing and walking. Am J Phys Med Rehabil 2003; 82:511-516.

-43 Winstein CJ, Gardner ER, McNeal DR, Barto PS, Nicholson DE: Standing balance training: effect on balance and locomotion in hemiparetic adults. Arch Phys Med Rehabil 1989;70:755-762.

44 Silsupadol P, Lugade V, Shumway-Cook A, van Donkelaar P, Chou LS, Mayr U, Woollacott $\mathrm{MH}$ : Training-related changes in dualtask walking performance of elderly persons with balance impairment: a double-blind, randomized controlled trial. Gait Posture 2009;29:634-639.

45 Granacher U, Mühlbauer T, Bridenbaugh S, Bleiker E, Wehrle A, Kressig RW: Balance training and multi-task performance in seniors. Int J Sports Med 2010;31:353-358. 